\title{
ADAPTACIÓN Y VALIDACIÓN ESPAÑOLA DEL CONTROLLING COACH BEHAVIORS SCALE (CCBS)
}

\author{
Rubén Trigueros-Ramos \\ Juan Miguel Fernández-Campoy \\ Antonio Alías \\ José Manuel Aguilar-Parra \\ Ma Carmen Lozano Segura \\ Departamento de Psicología Evolutiva y de la Educación \\ Universidad de Almería \\ E-mail: jmaguilar@ual.es
}

https://doi.org/10.17060/ijodaep.2017.n1.v2.954

Fecha de Recepción: 23 Marzo 2017

Fecha de Admisión: 1 Abril 2017

\section{RESUMEN}

El objetivo de este estudio fue validar y adaptar el Controlling Coach Behaviors Scale (CCBS). En el estudio participaron 444 estudiantes de secundaria con una edad media de 15.3 años. El análisis factorial confirmatorio reveló unos índices de ajuste adecuados para la nueva versión de la escala, mostrándose la estructura factorial invariante respecto al sexo. Los factores que integran la escala obtuvieron una alta consistencia interna y estabilidad temporal. El análisis de validez de criterio mostró que la intimidación y el control mediante recompensas predijeron significativamente la desmotivación, con un peso de regresión positivo. Este nuevo instrumento puede ayudar a analizar con validez y fiabilidad el estilo interpersonal de control del docente de educación física sobre los estudiantes.

Palabras clave: estilo interpersonal control, educación física, propiedades psicométricas

\section{ANTECEDENTES}

Uno de los objetivos principales de las clases de educación física (EF) es la consolidación de hábitos regulares de práctica de actividad física (AF), la ocupación activa del tiempo libre, ayudar a comprender los beneficios de la AF, y fomentar el desarrollo psíquico y social de los adolescentes. En este sentido, el profesor de EF puede tener una influencia significativa en la implicación de los estudiantes en las clases de EF, sin olvidarnos de las experiencias psicológicas que se derivan de su participación durante dichas clases (Chelladurai y Reimer, 1997).

Desde la Teoría de la Autodeterminación (TAD; Ryan y Deci, 2002) se sugiere que el comporta- 
miento del profesor se puede ver a través de dos estilos interpersonales: el apoyo a la autonomía y mediante conductas de control. Sin embargo, las investigaciones surgidas en los últimos años se han centrado principalmente en el apoyo a la autonomía por parte del docente (Moreno-Murcia y Huéscar, 2012; Moreno-Murcia, Huéscar Peco, Alarcón y Cervelló, 2013), mientras que apenas existen evidencias empíricas sobre el uso de comportamientos de control por parte del docente (Bartholomew, Ntoumanis, y Thøgersen-Ntoumani, 2010). Por ello, el propósito de este estudio es mostrar evidencias de validez y adaptar The Controlling Interpersonal Style in a Coaching Context: Development and Initial Validation of a Psychometric Scale con el fin de evaluar el estilo interpersonal control de los docentes en las clases de EF.

La TAD sugiere que el apoyo a la autonomía por parte del docente promueve activamente entre los estudiantes esfuerzos hacia la iniciativa propia, crea las condiciones para que experimenten un sentido de voluntad y selección propia y de autodesarrollo tanto a nivel mental como físico. Por ello, según las investigaciones surgidas desde la TAD, tanto a nivel internacional como nacional (Adie, Duda y Ntoumanis, 2012; Hagger, Chatzisarantis, Hein, Pihu, Soós y Karsai, 2007; Ferguson, Kasser y Jahng, 2011; Moreno-Murcia, Conde y Sáenz-López, 2012; Moreno, Hernández y González-Cutre, 2009), han demostrado que un estilo impersonal donde el docente fomente el apoyo a la autonomía está relacionado con las formas de motivación más autodeterminadas (eg, motivación intrínseca, regulación integrada y/o regulación identificada). En concreto, un estilo interpersonal de apoyo autonomía mejoraría la motivación más autodeterminada de los estudiantes, ya que contribuye a la satisfacción de las tres necesidades psicológicas como son la autonomía (el grado en que los individuos se sienten responsables del inicio de su comportamiento), la competencia (el grado en que los individuos son capaces de alcanzar sus objetivos deseados) y la relación con los demás (el grado en que las personas se sienten integrados en el grupo). Por el contrario, un estilo impersonal de control por parte del docente donde primen las presiones externas, tales como el uso de medios coercitivos, imposiciones, actuar de manera preconcebida, etc., son percibidos por los alumnos como el origen de sus comportamientos (Bartholomew, Ntoumanis, Ryan, Bosch y Thogersen-Ntoumani, 2011). Por ello, los alumnos experimentan un locus percibido de casualidad externo a ellos ya sea para evitar sentimientos de culpa (regulación introyectada), por demandas externas a ellos (regulación externa) o pierdan el completo interés por la actividad (desmotivación) (Sánchez-Oliva, Leo, Amado, Cuevas y García-Calvo, 2013).

Precisamente, resultan sorprendentes las pocas investigaciones que existen sobre el uso de estrategias de control por parte del docente. En este sentido, esta misma problemática está presente en la actividad física y el deporte. Por ello, partiendo de la misma necesidad de conocer la influencia de un estilo interpersonal de control por parte del entrenador sobre los atletas y deportistas Bartholomew, et al. (2010) crearon Controlling Coach Behaviors Scale (CCBS). Este instrumento contempla una definición multidimensional del control en actividad física, a partir de la revisión de este constructo en contextos similares. El CCBS fue desarrollado y validado originalmente por medio de cuatro estudios con muestras independientes de atletas.

En el primero de los estudios Bartholomew, et al. (2010), trataron de identificar las estrategias de entrenamiento de control de uso más frecuente durante los entrenamientos, a través de la respuesta dada por 17 atletas y 6 entrenadores a una serie de entrevistas individuales semiestructuradas y discusiones de grupo, además de la colaboración de expertos internacionales que colaboraron en la definición de las diferentes estrategias de control e idoneidad de los ítems. Al final del primer estudio se conformó una escala constituida por 33 ítems y cinco factores. Estos factores estaban integrados por: (a) Control mediante recompensas: consiste en el uso por parte de los entrenadores de recompensas extrínsecas y elogios para inducir la participación de atleta 0 de ciertos 
comportamientos; (b) Condicionamiento negativo: consiste en el uso de técnicas de retirada de atención 0 apoyo cuando no muestran los comportamientos deseados por el entrenador; (c) Intimidación: consiste en el uso de técnicas coercitivas y amenaza para conseguir la conducta deseada; (d) El control personal excesivo: refleja los comportamientos excesivamente intrusivos entrenadores; y por ultimo (e) Juzgar y menospreciar: consiste en el uso de comportamientos que tienen el objetivo de socavan activamente la autoestima de los atletas.

A partir de los 33 ítems y los cinco factores extraídas del estudio mixto, un segundo estudio de Bartholomew, et al. (2010) tenía por objetivo definir el cuestionario a través de un análisis factorial exploratorio para comprobar la composición factorial de los ítems generados en el primer estudio. Para ello, se valieron de una muestra compuesta por 264 de atletas (143 chicos y 115 chicas) entre 12 y 17 años. Así pues, teniendo en consideración los criterios de correlación inter-ítem y la correlación mínima corregida ítem-total, el cuestionario inicial formado por 33 ítems quedo reducido a 16 ítems (algunos de ellos reformulados) y además se añadió 6 nuevos ítems con el fin de aumentar las estimaciones de la fiabilidad interna y para que la estructura factorial del cuestionario pudiera ser probada en el estudio tres.

A continuación en el estudio tres, con el fin de refinar mas la estructura del cuestionario se realizo un análisis factorial confirmatorio (AFC) del cuestionario de 22 ítems. Un primer AFC mostró que los índices de ajuste del modelo eran aceptables, no obstante, eliminaron tres ítems, uno de excesivo control personal y dos de control mediante recompensas debido a que los ítems correlacionaban muy alto con otros ítems. Excluyendo estos tres ítems el ajuste del modelo mejoró. Sin embargo, las correlaciones de los factores revelaron que el factor de juzgar y menospreciar correlaciona altamente con tres de los otros cuatro factores (condicionamiento negativo, intimidación y el control personal excesivo).

Como resultado de las altas correlaciones, tomaron la decisión de eliminar el factor juzgar y menospreciar, resultando finalmente una escala compuesta por 15 ítems repartidos entre cuatro factores (cuatro para condicionamiento negativo, intimidación y control mediante recompensas y tres para control personal excesivo). El cuestionario presenta unos índices de ajuste apropiados con buenos índices de fiabilidad, además los factores correlacionaron de forma positiva. Adicionalmente, testaron un modelo de orden superior presentando unos índices de ajuste y fiabilidad apropiados, lo que indica que un estilo interpersonal de control es un constructo multidimensional separado, pero relacionado entre sí. En cuanto al último estudio, se realizo un análisis de invarianza respecto al sexo con el fin de testar si el cuestionario se muestra invariante respecto al género, para ello se valieron de una muestra de 189 sujetos dividido entre 50 chicos y 139 chicas con edades comprendida entre los 12 y 17 años. El cuestionario se mostró invariante respecto al género.

Asimismo, en España este instrumento fue validado y adaptado recientemente por Castillo, Tomás, Ntoumanis, Bartholomew, Duda y Balaguer (2014) a través de tres estudios. Para ello, se valieron de una muestra independiente para cada investigación. En el primero de ellos analizaron la estructura factorial del cuestionario, se valieron de una muestra de 373 futbolistas varones con edades comprendidas entre los 12 y 13 años, el cuestionario mostró una adecuada validez y fiabilidad. En el segundo y tercer estudio se valieron de 433 futbolistas varones con edades comprendidas entre los 12 y 15 años donde se analizó la estabilidad temporal y de 505 futbolistas de nivel regional y 145 de nivel autonómico para el análisis de invarianza según el nivel competitivo. Ambos análisis, mostraron unos índices adecuados de validez.

Partiendo de estudios previos que han analizado las conductas de control por parte del entrenador en el deporte, el objetivo del presente estudio es validar y adaptar el CCBS de Bartholomew, 
et al. (2010) al contexto español de las clases de EF. Con el fin de tener un instrumento adecuado para medir las percepciones de estilo interpersonal de control de los profesores de EF sobre los estudiantes. Para ello, se analizará la nueva estructura factorial del instrumento (CCBS), Ios estadísticos descriptivos y su invarianza por sexo, además de su consistencia interna y estabilidad temporal. También se analizó la validez de criterio de la nueva escala, comprobando las relaciones de los diferentes tipos de control sobre la desmotivación, las formas de motivación no autodeterminadas y las autodeterminadas. Con el fin de dotar de un instrumento que muestre evidencias de fiabilidad y validez para medir estilo interpersonal de control de los profesores de EF.

\section{MÉTODO}

\section{Participantes}

En el presente estudio tomaron parte 444 estudiantes de educación secundaria obligatoria y post-obligatoria (218 chicos y 216 chicas), con edades comprendidas entre los 13 y 19 años ( $M=$ 15.3; DT = 1.17), pertenecientes a dos centros educativos de la provincia de Almería.

Para analizar la estabilidad temporal del CCBS se utilizó una segunda muestra independiente de 52 estudiantes de educación post-obligatoria, con edades comprendidas entre 15 y 19 años $(M=$ $15.7 ; D T=0.78$ ), quienes completaron el instrumento en dos ocasiones, con un intervalo de tiempo de dos semanas entre la primera y segunda toma de datos

\section{Medidas}

Apoyo al control en el contexto de la EF. Con el fin de medir el apoyo al control en el contexto de las clases de EF por parte del docente, se validó y adaptó Controlling Coach Behaviors Scale (CCBS) de Bartholomew, et al. (2011). Este cuestionario está encabezado por la sentencia "En mis clases de Educación Física..." y consta de 15 ítems repartidos en cuatro factores, que miden el control mediante recompensas (eg." Mi profesor de EF trata de motivarme prometiéndome recompensas si lo hago bien"), condicionamiento negativo (eg. "Mi profesor de EF se muestra menos amigable conmigo cuando no hago el esfuerzo de ver las cosas como quiere"), la intimidación (eg." Mi profesor de EF me grita delante de otros para que haga algunas cosas") y excesivo control personal (eg." Mi profesor de EF espera que las clases de educación física sean el centro de mi vida"). Los estudiantes debían indicar su respuesta por medio de una escala Likert de 1 (nada verdadero) a 7 (totalmente verdadero).

\section{Procedimiento}

Con el objetivo de poder llevar a cabo la validación del cuestionario al contexto español se utilizó la estrategia de traducción inversa (Hambleton, 1996). Este proceso consiste en la traducción al español del cuestionario original realizado por un grupo de traductores expertos y posteriormente otro grupo lo tradujo a su idioma original. La bondad de la traducción se juzgó en función del grado de coincidencia con la versión original. La versión obtenida fue analizada por tres expertos (Lynn, 1986) en EF, de tal modo que se garantizara que los ítems obtenidos, estuvieran bien diseñados para medir el constructo que quería medirse, sin perder el significado original.

Una vez obtenido el cuestionario se contactó con los responsables de los centros educativos participantes, que a su vez derivaron en el profesorado de EF, a los que también se les informó del objetivo de la investigación y solicitó su colaboración. Al alumnado menor de edad se le requirió una autorización de sus padres para su participación. Antes de administrar la escala a la totalidad de los participantes, ésta fue cumplimentada por un pequeño grupo de alumnos para asegurar la correcta comprensión de todos los ítems. La administración del cuestionario se realizó bajo la supervisión 
de un encuestador experto miembro del grupo de investigación, que explicó y solventó las dudas que surgieron al cumplimentarlo. El tiempo estimado para completar el cuestionario estuvo en torno a 15 minutos.

\section{ANÁLISIS DE DATOS}

Con el fin de poder determinar la validez y fiabilidad de la CCBS en el contexto español se analizó las propiedades psicométricas del cuestionario. En primer momento, se realizó un análisis factorial confirmatorio (AFC) con el fin de testar la estructura factorial del cuestionario además de la estructura de un modelo que englobara el control como factor de orden superior. En segundo lugar, se realizó un análisis multigrupo para analizar la invarianza respecto al género con el fin determinar si el cuestionario es entendido por igual tanto por los chicos como por las chicas. Posteriormente, se llevo a cabo el análisis estadístico-descriptivos y el análisis de consistencia interna a través de alfa de Cronbach con el fin, este último, de testar la fiabilidad del instrumento y un análisis de estabilidad temporal (índice de correlación intra-clase, $\mathrm{CCI}$ ). Por último, se analizó la validez de criterio de los cuatro factores que integran la CCBS para ello, se realizó un análisis de regresión lineal para predecir la desmotivación en las clases de EF. Para los análisis de datos se utilizaron los paquetes estadísticos SPSS 19.0 y AMOS 19.0.

Debido a que el coeficiente de Mardia resulto ser alto (119.391) para el AFC se utilizó el método de estimación de máxima verosimilitud junto con el procedimiento de bootstrapping. Los estimadores no resultaron afectados por la falta de normalidad, por lo que se considerados robustos (Byrne, 2001). Con el objetivo de aceptar o rechazar el modelo testado, se tuvo en consideración un conjunto de índices de ajuste: 2/gl, CFI (Comparative Fit Index), TLI (Tucker Lewis Index), IFI (Incremental Fit Index), RMSEA (Root Mean Square Error of Approximation) más su intervalo de confianza (IC) al 90\%, y SRMR (Standardized Root Mean Square Residual). Dado que el 2 es muy sensible al tamaño de la muestra (Jöreskog y Sörbom, 1993), se empleó el 2/gl, considerándose aceptables valores inferiores a 5 (Bentler, 1989). Los índices incrementales (CFI, TLI e IFI) muestran un buen ajuste con valores iguales o superiores .90 (Schumacker y Lomax, 1996), mientras que los índices de error (RMSEA y SRMR) se consideran aceptables con valores iguales o menores de 08 (Browne y Cudeck, 1993; Hu y Bentler, 1999).

\section{RESULTADOS}

\section{Análisis factorial confirmatorio}

Los índices de ajuste del modelo testado (Figura 1) reveló unos índices de ajuste apropiados: $\chi^{2}(84 . N=444)=323.41, p<.001 ; \chi^{2} / g l=3.85 ; \mathrm{CFI}=.94 ; \mathrm{TLI}=.93 ; \mathrm{IFI}=.94 ; \mathrm{RMSEA}=.080$ (IC $90 \%=.071-.089) ; \mathrm{SRMR}=.045$. Los pesos de regresión estandarizados oscilaron entre .60 y .84 siendo estadísticamente significativos $(p<.001)$. En cuanto a las correlaciones entre los factores estos oscilaron entre .70 y .85 siendo estadísticamente significativos $(p<.001)$. 


\section{ADAPTACIÓN Y VALIDACIÓN ESPAÑOLA DEL CONTROLLING COACH BEHAVIORS SCALE (CCBS)}

Figura 1.

Análisis factorial confirmatorio del CCBS. Las elipses representan los 3 factores y los rectángulos los diferentes ítems. Las varianzas residuales se muestran en 4 los círculos pequeños.

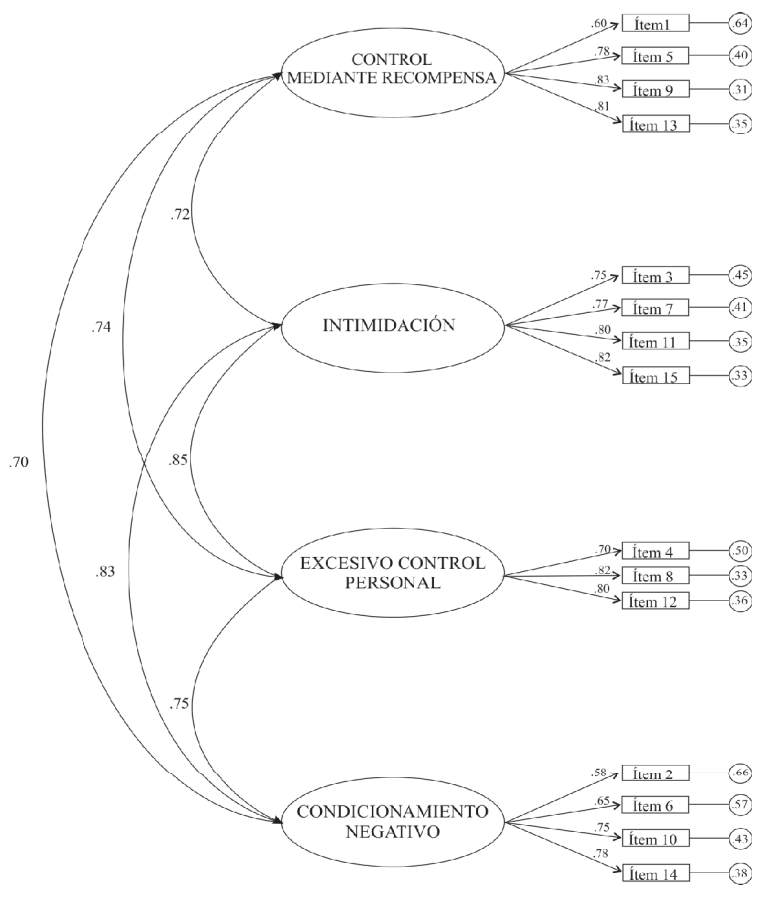

Una vez determinado el modelo, se procedió a testar un modelo de orden superior (eg., los cuatro factores de primer orden convergiendo en un factor de segundo orden superior denominado control). Los índices de ajuste de este modelo fueron ligeramente mejores, aunque en general aceptables: $\chi 2(86 . \mathrm{N}=444)=331.60, p<.001 ; \chi 2 / \mathrm{gl}=3.86 ; \mathrm{CFI}=.94 ; \mathrm{TLI}=.93 ; \mathrm{IFI}=.94 ; \mathrm{RMSEA}=$ $.080($ IC $90 \%=.071-.090)$; SRMR $=.044$. Todos los pesos de regresión estandarizados fueron significativos $(p<.001)$, siendo de .80 para control mediante recompensas, .87 para condicionamiento negativo, .95 para intimidación, .90 excesivo control personal.

\section{Análisis de invarianza por sexo}

Para saber si la estructura factorial del modelo se muestra invariante respecto al género, se realizo un análisis multigrupo. Tal y como muestra la tabla 1 no se encontraron diferencias significativas en el estadístico 2 entre el modelo no constreñido (Modelo 1) y el modelo con pesos de medida invariante (Modelo 2) y si entre los modelos de covarianzas estructurales invariantes (Modelo 3), y medidas residuales invariantes (Modelo 4). En la tabla 1 también se muestran los diversos índices de ajuste para los seis modelos comparados dentro de la estructura de un factor de orden superior. Igualmente, se encontraron diferencias significativas entre el modelo no constreñido (Modelo 1) y el modelo con pesos de medidas invariantes (Modelo 2) y si hubo diferencias significativas entre los 
modelo de pesos estructurales invariantes (Modelo 3), covarianzas estructurales (Modelo 4), residuales estructurales invariantes (Modelo 5), y medidas residuales invariantes (Modelo 6). Las diferencias entre ambos modelos (modelo 1 y modelo 2) constituye un criterio mínimo para afirmar que la estructura factorial del cuestionario, modelo de cuatro factores y el modelo de orden superior, se muestra invariante respecto al género.

Tabla 1

Análisis de Invarianza por Sexo

\begin{tabular}{lllllllllll}
\hline \multicolumn{1}{l}{ Modelo de cuatro factor de orden primario } \\
\hline Modelos & $\chi^{2}$ & $g l$ & $\chi^{2} / g l$ & $\Delta \chi^{2}$ & $\Delta g l$ & CFI & TLI & IFI & RMSEA & SRMR \\
\hline Modelo 1 & 442,79 & 168 & 2,64 & - & - &, 93 &, 92 &, 93 &, 061 &, 047 \\
Modelo 2 & 462,37 & 179 & 2,58 & 19,58 & 11 &, 93 &, 92 &, 93 &, 060 &, 049 \\
Modelo 3 & 481,37 & 189 & 2,55 & $38,59^{*}$ & 21 &, 93 &, 92 &, 93 &, 059 &, 076 \\
Modelo 4 & 514,99 & 204 & 2,52 & $72,20^{* * *}$ & 36 &, 92 &, 92 &, 92 &, 059 &, 080 \\
\hline
\end{tabular}

\begin{tabular}{lccccccccccc}
\hline \multicolumn{1}{l}{ Modelo de un factor de orden superior } \\
\hline Modelos & $\chi^{2}$ & $g l$ & $\chi^{2} / g l$ & $\Delta \chi^{2}$ & $\Delta g l$ & CFI & TLI & IFI & RMSEA & SRMR \\
\hline Modelo 1 & 452,06 & 172 & 2,53 & - & - &, 93 &, 92 &, 93 &, 061 &, 048 \\
Modelo 2 & 471,68 & 183 & 2,58 & 19,62 & 11 &, 93 &, 92 &, 93 &, 060 &, 050 \\
Modelo 3 & 472,42 & 186 & 2,56 & $24,35^{*}$ & 14 &, 93 &, 92 &, 93 &, 059 &, 054 \\
Modelo 4 & 483,75 & 187 & 2,59 & $31,68^{* *}$ & 15 &, 93 &, 92 &, 93 &, 060 &, 075 \\
Modelo 5 & 489,66 & 191 & 2,56 & $37,59^{* *}$ & 19 &, 92 &, 92 &, 92 &, 059 &, 076 \\
Modelo 6 & 522,70 & 206 & 2,54 & $70,64^{* * *}$ & 34 &, 92 &, 92 &, 92 &, 059 &, 083 \\
\hline
\end{tabular}

$* p<, 05 ; * * p<, 01 ; * * * p<, 001$.

\section{Estadísticos descriptivos, análisis de correlación y de fiabilidad}

Tal y como muestra la Tabla 2 los participantes en el estudio obtuvieron una mayor puntuación media para el condicionamiento negativo 2.47 y una menor puntuación para intimidación 1.85.

Para obtener evidencias de la fiabilidad de la escala se realizó un análisis de consistencia interna y de estabilidad temporal de los cuatro factores (ver Tabla 2). El análisis de consistencia interna reveló valores alfa de Cronbach que oscilaron entre .79 y .86. Para el análisis de estabilidad temporal, se calcularon los coeficientes de correlación intra-clase (CCI) y sus intervalos de confianza (IC). Los resultados oscilaron entre .80 y .85 para cada una de las subescalas de la ECCPEF.

En cuanto al análisis de consistencia interna del modelo de orden superior, la puntuación global de control fue de .93 y el análisis de estabilidad temporal .90 (CCI) y .89 (IC). 
ADAPTACIÓN Y VALIDACIÓN ESPAÑOLA DEL CONTROLLING COACH BEHAVIORS SCALE (CCBS)

Tabla 2

Estadísticos Descriptivos, Alfa de Cronbach, Correlaciones Bivariadas y Análisis de Estabilidad Temporal

\begin{tabular}{lcccccccc}
\hline Factores & $M$ & $D T$ & $\alpha$ & 1 & 2 & 3 & 4 & CCI \\
\hline 1. Control Mediante Recompensas & 2.44 & 1.05 & .84 & & $.63 * * *$ & $.64 * * *$ & $.58^{* * *}$ & .80 (IC $=.77-.85)$ \\
2. Intimidación & 1.89 & 1.14 & .89 & & $.74 * * *$ & $.70^{* * *}$ & $.85(\mathrm{IC}=.80-.87)$ \\
3. Excesivo control personal & 2.04 & 1.10 & .83 & & & $.63^{* * *}$ & $.84(\mathrm{IC}=.87-.81)$ \\
4. Condicionamiento Negativo & 2.48 & 1.10 & .81 & & & & $.82(\mathrm{IC}=.78-.85)$
\end{tabular}

$* * * p<, 001$

\section{Análisis de validez de criterio}

Con el fin de analizar la validez de criterio del CCBS se realizó un análisis de regresión lineal por el cual se introdujo como variable dependiente la desmotivación, las motivaciones no autodeterminadas y las motivaciones autodeterminadas y los diferentes tipos de control como variables independientes.

Los resultados (Tabla 3) revelaron que la intimidación y el control mediante recompensas predice de forma significativa la desmotivación, con un peso de regresión positivo y proporcionando una varianza explicada del $18 \%$. A su vez, el condicionamiento negativo y el control mediante recompensas predicen de forma significada las formas de motivación menos autodeterminadas, con un peso de regresión positivo y proporcionando una varianza explicada del $11 \%$. Por último, la intimidación predice de forma significativa las formas de motivación más autodeterminadas, con un peso de regresión negativo y proporcionando una varianza explicada del 7\%.

Tabla 3

Análisis de Regresión Lineal de las Formas de Conductas de Control del Profesor que Predicen la desmotivación, las motivaciones no autodeterminadas y las autodeterminadas

\begin{tabular}{|c|c|c|c|c|c|c|c|c|c|c|c|c|}
\hline \multirow[b]{2}{*}{ Variables } & \multicolumn{4}{|c|}{ Desmotivación } & \multicolumn{4}{|c|}{ No autodeterminadas } & \multicolumn{4}{|c|}{ Autodeterminadas } \\
\hline & $F$ & $R^{2}$ & $\beta$ & $t$ & $F$ & $R^{2}$ & $\beta$ & $t$ & $F$ & $R^{2}$ & $\beta$ & $t$ \\
\hline & 24,07 &, $18^{* * *}$ & & & 13,78 &, $11 * * *$ & & & 8,12 &, $07 * * *$ & & \\
\hline 1. Control Mediante Recompensas & & &, 16 & $2,67 * *$ & & &, 14 & $2,20 * *$ & & &, 04 &, 59 \\
\hline 2. Intimidación & & &, 30 & $4,14 * * *$ & & &, 12 & 1,54 & & &,- 32 & $-4,13 * * *$ \\
\hline 3. Excesivo control personal & & &, 04 & ,60 & & &,- 02 & $-0,27$ & & &, 08 & 1,12 \\
\hline 4. Condicionamiento Negativo & & &,- 04 &,- 70 & & &, 15 & $2,22 * *$ & & &,- 03 &,- 38 \\
\hline
\end{tabular}

\section{DISCUSIÓN}

El objetivo del presente estudio es validar y adaptar la CCBS de Bartholomew, et al. (2010). Los resultados han mostrado que es un instrumento que muestra evidencias de validez y fiabilidad para medir los diferentes aspectos conductuales de estilo interpersonal control del profesor, presentando correlaciones positivas entre los diferentes factores que integran el cuestionario. Este instrumento permitirá analizar con más profundidad la interactuación del docente con sus alumnos mostrando evidencias empíricas que posteriormente puedan relacionarse con la implicación de los estu- 
diantes en las clases de EF, adopción de estilos de vida saludables y una práctica más activa del tiempo libre.

Los resultados del AFC revelaron unos índices de ajuste bastante aceptables, mostrando unas correlaciones positivas entre los factores. En cuanto al análisis de fiabilidad, hay que destacar que se obtuvieron valores aceptables de estabilidad temporal (Cicchetti, 1994), junto con valores de consistencia interna por encima de .80 para todos los factores. Además se mostró invariante respecto al género, por lo que resulta interesante en la medida en que permiten en el futuro utilizar el CCBS para realizar comparaciones del control percibido en EF respecto al género.

Estos resultados pueden resultar importantes ya que ayudaría a comprender y profundizar en la teoría de la autodeterminación. En el sentido de que, en investigaciones donde el foco de estudio sea las experiencias psicológicas de los estudiantes de EF, el estilo interpersonal control del docente, predecirá un malestar y una mala adaptación a las clases de EF (Deci y Ryan, 2000; MorenoMurcia, Huéscar, y Cervelló, 2012; Ntoumanis, 2012). Esto se debe a que las estrategias de control tienen la capacidad de frustrar los sentimientos de autonomía, competencia y relación con los demás (Bartholomew, Ntoumanis, y Thøgersen-Ntoumani, 2011). En este sentido, los comportamientos excesivamente intrusivos, intentar controlar y restringir determinadas conductas tanto en la vida de los estudiantes como durante las clases y el mal uso de las recompensas puede llevar a sentimientos de resentimiento hacia el docente y provocar en el alumnado falta de confianza, incapacidad para autoregular sus propios comportamientos e animadversión hacia las clases de EF y la actividad física.

Los resultados del AFC apoyaron también la estructura de un modelo de orden superior que representa el control percibido en EF. Desde el punto de vista metodológico este modelo apoya el uso de la medida global del control a partir de la media de sus cuatro dimensiones. Esta medida global puede ser interesante en modelos complejos donde se necesite relacionar el control percibido en EF con otras variables.

Hay que mencionar que aunque los resultados de este estudio proporcionan apoyo psicométrico para el CCBS, existen algunas limitaciones que deben ser indicadas. Primeramente, la muestra del estudio ha sido no probabilística y, por tanto, los resultados no pueden ser generalizados a otros grupos de estudiantes. Asimismo, el proceso de validación del instrumento debe de ser continuado la validación y por tanto futuros trabajos deberían de explorar la estructura factorial de la escala con muestras diferentes de estudiantes (ej. edad, nivel educativo, ubicación física). Segundamente, el análisis multigrupo ha mostrado evidencias de que el instrumento puede ser utilizado para comparar el control percibido en las clases de EF entre chicos y chicas, no obstante, futuros trabajos deberían determinar si también puede ser utilizado para establecer diferencias en el control percibido en las clases de EF en función de otras variables (ej. tipo de centro, nivel educativo). Por último, con el fin de mostrar evidencias de la validez predictiva del instrumento los futuros trabajos podrían considerar variables que actúen de antecedentes o consecuentes del control percibido en las clases de EF, y analizar la asociación del constructo con estas variables.

En resumen, los resultados de este estudio apoyan el CCBS como un instrumento válido y fiable para medir de forma multidimensional el control percibido en las clases de EF. Bartholomew, et al. (2010) creó el CCBS como un instrumento multidimensional diseñado para medir el control percibido por los atletas en el contexto deportivo desde la perspectiva de la TAD. Sin embargo, a través de este estudio se ha mostrado evidencias de que el instrumento puede también ser utilizado para valorar el control percibido por los estudiantes en el contexto de la EF escolar. De este modo, el profesorado y directores de centros educativos en España puedan utilizar la escala para autoreflexionar sobre las estrategias de motivación que se emplean durante las clases de EF. 


\section{REFERENCIAS}

Adie J., Duda J.L. y Ntoumanis N. (2012) Perceived coach autonomy support, basic need satisfaction and the well- and ill-being of elite youth soccer players: a longitudinal investigation. Psychology of Sport and Exercise, 13, 51-59.

Bartholomew, K.J., Ntoumanis, N., y Thøgersen-Ntoumani, C. (2010). The controlling interpersonal style in a coaching context: Development and initial validation of a psychometric scale. Journal of Sport and Exercise Psychology, 31, 193-216.

Bartholomew, K., Ntoumanis, N., y Thøgersen-Ntoumani, C. (2011). Self-Determination theory and the darker side of athletic experience: The role of interpersonal control and need thwarting. Sport and Exercise Psychology Review, 7, 23-27.

Bartholomew, K., Ntoumanis, N., Ryan, R., Bosch, J., y Thogersen-Ntoumani, C. (2011). SelfDetermination theory and diminished functioning: The role of interpersonal control and psychological need thwarting. Personality and Social Psychology Bulletin, 37, 1459-1473.

Bentler, P. M. (1989). EQS structural equations program manual. Los Angeles: BMDP Statistical Software.

Browne, M. W. y Cudeck, R. (1993). Alternative ways of assessing model fit. En K. Bollen y J. Long (Eds.), Testing structural equation models (pp. 136-162). Newbury Park, CA: Sage.

Byrne, B. M. (2001). Structural equation modeling with Amos: Basic concepts, applications and programming. Mahwah, NJ: Erlbaum.

Castillo, I., Tomás, I., Ntoumanis, N., Bartholomew, K., Duda, J. L., y Balaguer, I. (2014). Propiedades psicométricas de la versión española de la Escala de Conductas Controladoras del Entrenador en el contexto deportivo. Psicothema, 26(3), 409-414.

Chelladurai, P. y Riemer, H. A. (1997). A classification of the facets of athlete satisfaction. Journal of Sport Management, 11, 133-159.

Deci, E. L., y Ryan, R. M. (2000). The "what" and "why" of goal pursuits: Human needs and the selfdetermination of behavior. Psychological Inquiry, 11, 227-268.

Ferguson, Y. L., Kasser, T., y Jahng, S. (2011). Differences in life satisfaction and school satisfaction among adolescents from three nations: The role of perceived autonomy support. Journal of Research on Adolescence, 21, 649-661.

Hagger, M.S., Chatzisarantis, N.L.D., Hein, V., Pihu, M., Soós, I., y Karsai, I. (2007). The perceived autonomy support scale for exercise settings (PASSES): Development, validity and cross-cultural invariance in young people. Psychology of Sport and Exercise, 8, 632-653.

Hambleton, R. K. (1996). Adaptación de tests para su uso en diferentes idiomas y culturas: fuentes de error, posibles soluciones y directrices prácticas. En J. Muñiz (Ed.), Psicometría (pp. 207238). Madrid: Universitas.

Hu, L. y Bentler, P. M. (1999). Cutoff criteria for fit indexes in covariance structure analysis: Conventional criteria versus new alternatives. Structural Equation Modeling, 6, 1-55.

Jöreskog, K. G. y Sörbom, D. (1993). LISREL 8: Structural equation modeling with the SIMPLIS command language. Chicago, IL: Scientific Software International Inc.

Lynn, M. R. (1986). Determination and quantification of content validity. Nursing Research, 35, 382385.

Moreno-Murcia, J. A., Conde, C., y Sáenz-López, P. (2012). Importancia del apoyo de autonomía en la figura del docente en educación física. Tándem, 40, 18-27.

Moreno-Murcia, J. A., y Huéscar, E. (2012). Relación del tipo de feed-back del docente con la percepción de autonomía del alumnado en clases de educación física. Infancia y Aprendizaje, 35(1), 87-98. 
Moreno-Murcia, J. A., Huéscar, E., y Cervelló, E. (2012). Prediction of adolescents doing physical activity after completing secondary education. Spanish Journal of Psychology, 15, 90-100.

Moreno-Murcia, J. A., Huéscar, E., Peco, N., Alarcón, E., y Cervelló, E. (2013). Relación del feed-back y las barreras de comunicación del docente con la motivación intrínseca de estudiantes adolescentes de educación física. Anales de psicología, 1(29), 257-263.

Ntoumanis, N. (2012). A self-determination theory perspective on motivation in sport and physical education: Current trends and possible future research directions. En G . C. Roberts y D. C. Treasure (Eds.), Motivation in sport and exercise: Volumen 3 (pp. 91-128). Champaign, IL: Human Kinetics.

Ryan, R.M., y Deci, E.L. (2002). Overview of self-determination theory: An organismic dialectical perspective. In E.L. Deci y R.M. Ryan (Eds.), Handbook of self-determination research (pp. 333). Rochester, NY: University of Rochester Press.

Sánchez-Oliva, D., Leo, F. M., Amado, D., Cuevas, R., y García-Calvo, T. (2013). Desarrollo y validación del cuestionario de apoyo a las necesidades psicológicas básicas en educación física. Motricidad. European Journal of Human Movement, 30, 53-71.

Schumacker, R. E. \& Lomax, R. G. (1996). A beginner's guide to structural equation modeling. Mahwah, NJ: Erlbaum.

\section{APÉNDICE}

The Controlling Coach Behaviors Scale (CCBS)

\section{Control mediante recompensas}

1. Mi profesor trata de motivarme mediante recompensas si hago bien los ejercicios

5. Mi profesor sólo me premia o alaba para que me esfuerce en clase

9. Mi profesor sólo utiliza recompensas/alabanza para mantener la concentración durante las clases.

13. Mi profesor utiliza recompensas/alabanza para que complete todos los ejercicios que él/ella establece en clase

\section{Condicionamiento negativo}

2. Mi profesor se muestra menos amigable conmigo si no me esfuerzo hacer los ejercicios

6. Mi profesor es menos favorable conmigo cuando no estoy haciendo los ejercicios y esforzándome lo suficiente.

10. Mi profesor me presta menos atención si se siente disgustado conmigo

14. Mi profesor me ignora si él/ella se siente decepcionado conmigo

\section{Intimidación}

3. Mi profesor me grita delante de otros para obligarme a hacer los ejercicios

7. Mi profesor me suele amenazar con castigarme para que realice los ejercicios

11. Mi profesor me intimida para que realice los ejercicios que él/ella quiere que yo haga

15. Mi profesor me avergüenza delante de los demás, si realizo los ejercicios que él/ella quiere que yo haga

\section{Excesivo control personal}

4. Mi profesor espera que toda mi vida gire en torno a las clases de educación física

8. Mi profesor trata de controlar lo que hago en mi tiempo libre.

12. Mi profesor trata de interferir en los aspectos de mi vida fuera de las clases de Educación Física. 
